\title{
$\gamma \rightarrow \varepsilon$ martensite transformation and twinning deformation in fcc cobalt during surface mechanical attrition treatment
}

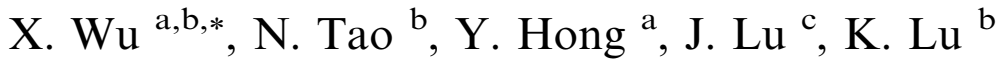 \\ ${ }^{a}$ State Key Laboratory of Nonlinear Mechanics, Institute of Mechanics, Chinese Academy of Sciences, Beijing 100080, China \\ b Shenyang National Laboratory for Materials Sciences, Institute of Metal Research, Chinese Academy of Sciences, Shenyang 110016, China \\ ${ }^{\mathrm{c}}$ LASMIS, University of Technical of Troyes, 10000 Troyes, France
}

Received 2 November 2004; accepted 2 December 2004

Available online 25 December 2004

\begin{abstract}
The deformation microstructure of face-centered cubic cobalt subjected to surface mechanical attrition treatment was studied as a function of strain levels. Strain-induced $\gamma \rightarrow \varepsilon$ transformation and twinning deformation were evidenced by transmission electron microscopy and were found to progress continuously in ultrafine and nanocrystalline grains as the strain increased.

(C) 2004 Acta Materialia Inc. Published by Elsevier Ltd. All rights reserved.
\end{abstract}

Keywords: Martensite transformation; Twinning; Nanostructure; Surface mechanical attrition treatment; Cobalt

\section{Introduction}

Polycrystalline cobalt usually presents a combination of $\varepsilon$ hexagonal close-packed (hcp) and $\gamma$ face-centered cubic (fcc) phases at room temperature [1]. The $\gamma$ phase is metastable at room temperature and will experience a strain-induced $\gamma \rightarrow \varepsilon$ martensite transformation on deformation $[1,2]$. X-ray diffraction analyses have demonstrated this phenomenon in ball-milled nanocrystalline $(\mathrm{nc})$ cobalt $[3,4]$. In particular, the response of $\gamma \rightarrow \varepsilon$ transformation to deformation can lead to enhanced properties, e.g. greater work hardening and higher tensile ductility, as often observed in coarsegrained metastable alloys $[5,6]$. On the other hand, $\gamma$ cobalt with low stacking fault energy facilitates twinning on deformation [3]. Twinning may play a crucial role in enhancing work hardening due to the fact that twin boundaries act as strong barriers to dislocation slipping [7-9]. Twinning has been demonstrated recently as being \footnotetext{
1284.

${ }^{*}$ Corresponding author. Tel.: +86 106261 8150; fax: +86 106256

E-mail address: xlwu@imech.ac.cn (X. Wu).
}

the deformation mechanism in nc metals [10-12]. Hence, the deformation microstructure of $\gamma$ cobalt may be quite complex due to the deformation mechanism involving interplay between slip, the phase transformation potential and twinning induced by deformation. In the present study the detailed characteristics of deformation microstructure of the $\gamma$ phase in cobalt subjected to surface mechanical attrition treatment (SMAT) [13] is investigated and correlated with the simultaneous occurrence of $\gamma \rightarrow \varepsilon$ transformation and twinning.

\section{Experimental procedure}

The material used in this study was an electrodeposited cobalt plate (purity: $99.98 \mathrm{wt} \%$ ). The X-ray diffraction analysis indicated a duplex hcp and fcc (volume fraction $\sim 18 \%$ ) structure of the product. The average grain size was found to be $\sim 30 \mu \mathrm{m}$.

The technique of SMAT was described in detail in our previous papers [13,14]. In brief, during the SMAT process, the hardened steel balls of $8 \mathrm{~mm}$ in diameter were placed at the bottom of a cylinder-shaped vacuum chamber attached to a vibration generator, with which 
the balls were resonated. Because of the high vibration frequency of the system, the sample surface was peened repetitively by a large number of balls within a short period of time. As a consequence the grains in the treated layer of the sample were effectively refined and revealed gradient distribution of grain sizes due to the gradient of strain varying from the surface (extremely large) towards the deep matrix (essentially zero) [13]. Hence, the deformation microstructure of various grain size regimes could be examined at different levels of strain. In the present work, the SMAT process was performed for $50 \mathrm{~min}$ at room temperature with a vibrating frequency of $50 \mathrm{~Hz}$ in a vacuum. The present study focused on the fcc phase in cobalt while a study of the hcp phase has been reported elsewhere [15].

After SMAT had been carried out, the microstructure was characterized using a transmission electron microscope (TEM, JEM200CX) operated at $200 \mathrm{kV}$. Both cross-sectional and plane foils were prepared [15].

\section{Results}

The deformation microstructure of the $\gamma$ phase was first studied at a low strain level. Fig. 1(a) shows the intersecting planar arrays of dislocations due to slipping of dislocations on respective $\{111\}$ plane at a depth of $\sim 170 \mu \mathrm{m}$ deep below the treated surface. Fig. 1(b) shows $\{111\}$ deformation twins $(\sim 160 \mu \mathrm{m}$ deep). Such deformation modes are characteristic of fcc metals with low stacking fault energy.

Fig. 2(a) and (b) shows the presence of lamellae in the $\gamma$ phase at higher strain $(\sim 150 \mu \mathrm{m}$ deep). According to the electron diffraction pattern (EDP) as shown in Fig. 2(c), the composite diffractions of the fcc, the $\{111\}$ twin, and hep are identified. Hence, the lamellae consist of the stacking of platelets of $\gamma$, twin, and martensite platelets. This indicates the onset of the $\gamma \rightarrow \varepsilon$ martensite transformation in the presence of strain. The $\gamma \rightarrow \varepsilon$ transformation results in largely coherent martensite platelets, having the $(0001)$ habit plane and strictly complying with the following orientation relationship,

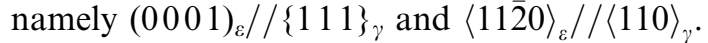

The platelets labeled $\mathrm{M}_{1}-\mathrm{M}_{6}$ in Fig. 2(a) are $\varepsilon$-martensites according to the EDP as shown in Fig. 2(d). It is interesting to note the presence of the basal stacking faults (SFs) in the martensites. Between the martensite platelets lies the $\gamma$ phase, as evidenced by the EDP shown in Fig. 2(e), but in this case it reveals a $\{111\}$ twin relationship. Deformation twins with two $\{111\}_{\gamma}$ orientations, indicated by solid and hollow triangles in Fig. 2(a) are visible in the $\gamma$ phase. The inset in Fig.
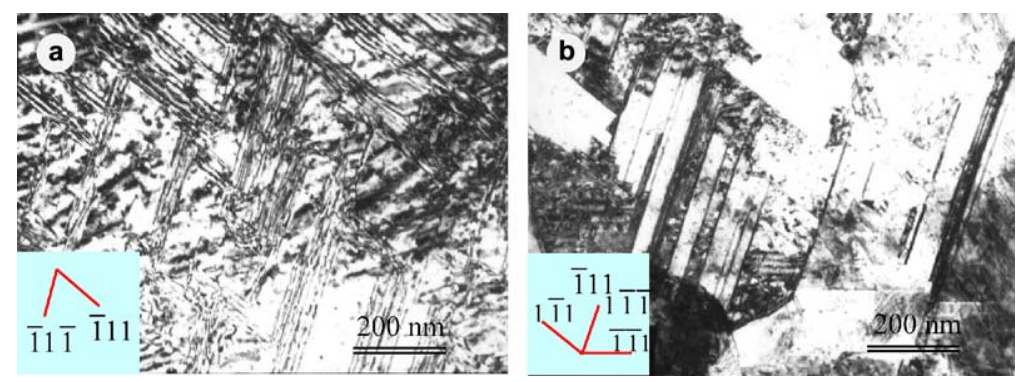

Fig. 1. (a) Planar arrays of dislocations $(\sim 170 \mu \mathrm{m}$ deep) and (b) deformation twins $(\sim 160 \mu \mathrm{m}$ deep).
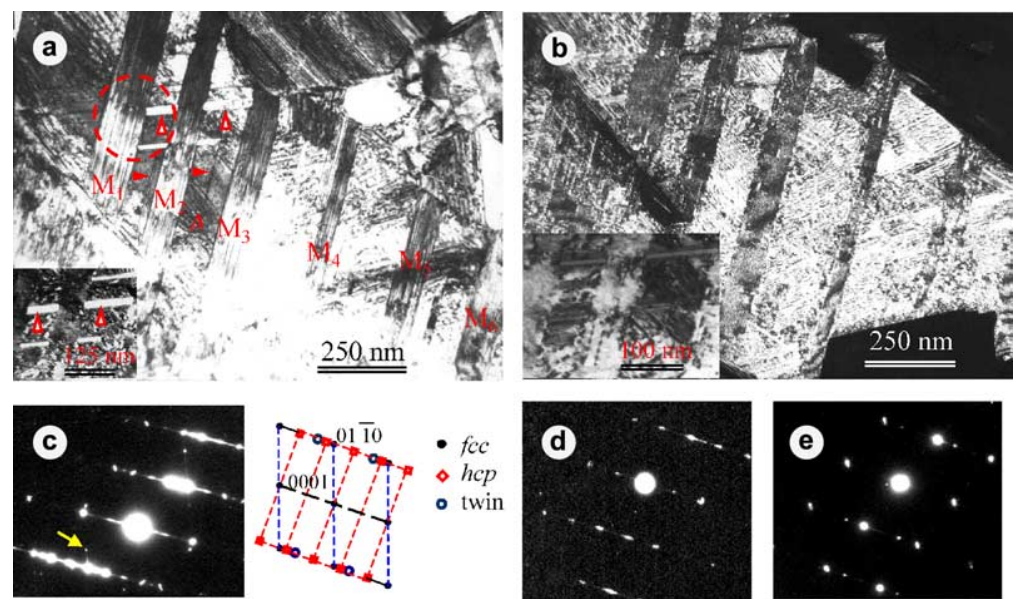

Fig. 2. (a) Lamellae in $\gamma$ phase $(\sim 150 \mu \mathrm{m}$ deep); (b) dark-field image of $\gamma$; (c) EDP from the encircled area in (a), with the zone axis $[\overline{1} 10]_{\gamma} / /[1 \overline{1} 0]_{\mathrm{T}} / /[01 \overline{1} 0]_{\varepsilon} ;$ (d) and (e) EDPs from $\mathrm{M}_{1}$ and $\mathrm{A}$ in (a), with [0110] and [110] zone axis, respectively. 
2(a) is the dark-field image of twins according to the spot indicated by an arrowhead in (c). In the dark-field image of the $\gamma$ phase shown in Fig. 2(b), it can be seen that the high density dislocation arrays lie in two $\{111\}_{\gamma}$ orientations. The inset shows dislocations in the third $\{111\}_{\gamma}$ orientation, forming pile-ups adjacent to twin boundaries. This indicates that both $\varepsilon$-martensite and twin boundaries may act as strong barriers to block dislocation. Plastic accommodation occurs preferentially in the $\gamma$ phase due to the formation of martensites.

The $\gamma \rightarrow \varepsilon$ transformation continues as the strain increases. Fig. 3(a) shows the formation of intersecting networks of $\varepsilon$-martensites on three of four possible sets of $\{111\}$ planes $(\sim 140 \mu \mathrm{m}$ deep). The martensites with an orientation of $T_{1}$ and $T_{2}$ occur in the $\gamma$ phase between the first set of $T_{3}$ orientated martensites. The high magnification dark field image in Fig. 3(b) shows $T_{1}$ orientated $\varepsilon$-martensites. Fig. 3(c) is the composite EDP taken from the encircled area, consisting of one fcc pattern with a [1 10$]$ zone axis superimposed on two hep patterns with [2 $\overline{1} \overline{1} 0]$ zone axis showing a $\{10 \overline{1} 1\}$ twin relationship.

As the strain increases, the $\gamma$ phase becomes significantly finer. Fig. 4(a) shows a submicron grain with many streaks inside $(\sim 80 \mu \mathrm{m}$ deep). Fig. 4(b) corresponds to a composite EDP. The $\gamma$, twin, and martensite can be clearly identified. This indicates that $\gamma \rightarrow \varepsilon$ transformation and deformation twinning have both taken place. Fig. 4(c) is the dark-field image of the twins.

Fig. 5(a) shows the formation of nc grains in the top surface layer where strain is significantly increased ( $\sim 40 \mu \mathrm{m}$ deep) [13]. The inset shows an EDP indicative of a combination of $\varepsilon$ and $\gamma$ phases. The lattice structure was determined and very marked in some of the grains. Most of the grains contain streaking. This may correspond to either SFs in the $\varepsilon$ phase or twins and martensites in the $\gamma$ phase. Fig. 5(b) is the dark-field image of an $\mathrm{nc}$ grain with a diameter of $\sim 90 \mathrm{~nm}(\sim 35 \mu \mathrm{m}$ deep $)$. The inset shows a composite EDP similar to that shown in Fig. 2(c), which indicates that $\gamma \rightarrow \varepsilon$ transformation and twinning deformation may occur simultaneously in nc grains. Fig. 5(c) is a lattice image (taken using high resolution $2010 \mathrm{~F}$ TEM) of an nc grain of $\sim 80 \mathrm{~nm}$ in diameter ( $\sim 20 \mu \mathrm{m}$ deep). The fcc and hcp crystal lattices
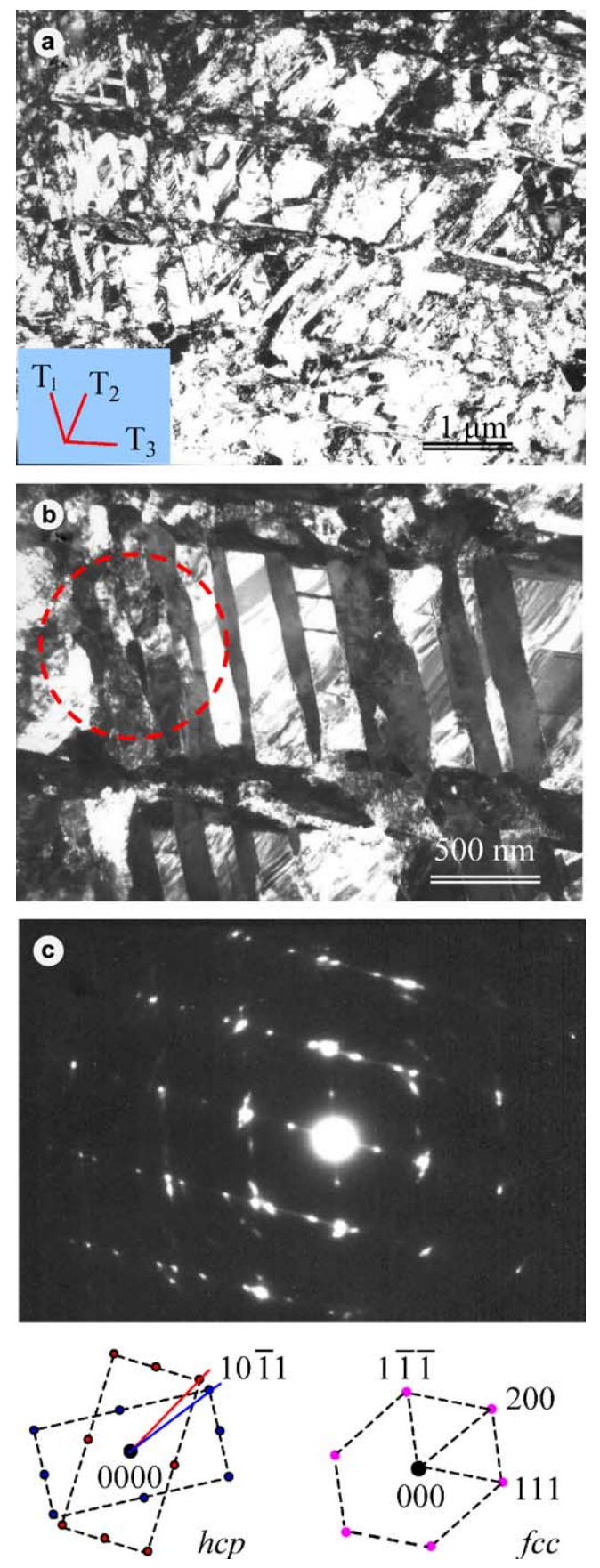

Fig. 3. (a) Intersecting networks of martensites ( $140 \mu \mathrm{m}$ deep); (b) high magnification dark-field of martensites and (c) EDP from the encircled area in (b).
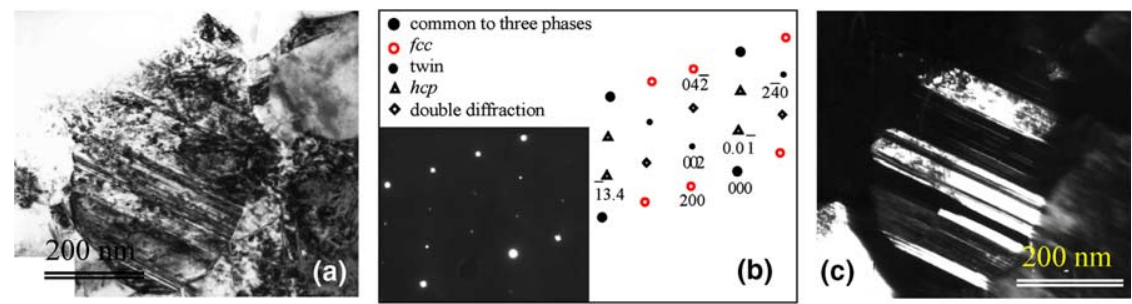

Fig. 4. (a) Bright-field image of an ultrafine grain ( $>0 \mu \mathrm{m}$ deep); (b) EDP with the zone axis $[012]_{\gamma} / /[210]_{\mathrm{T}} / /[\overline{4} 8 \overline{4} \overline{9}]_{\varepsilon}$ and (c) dark-field image of twins. 

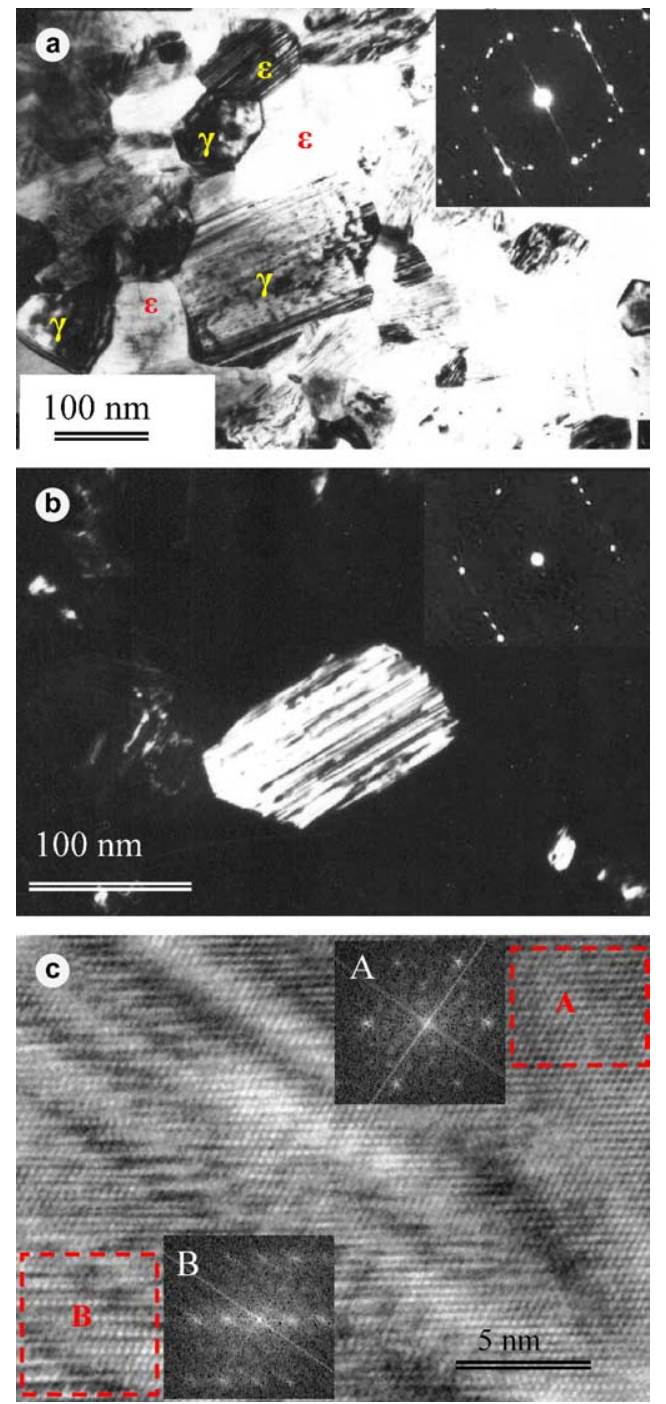

Fig. 5. (a) Nc grains ( $40 \mu \mathrm{m}$ deep); (b) dark-field image of an nc grain $(\sim 35 \mu \mathrm{m}$ deep) and (c) lattice image of an nc grain $(\sim 25 \mu \mathrm{m}$ deep). Insets are corresponding EDPs.

is indicated by squares $\mathrm{A}$ and $\mathrm{B}$, respectively. The insets (A) and (B) are corresponding fast Fourier transform (FFT) EDPs, with the zone axes [110] and [21̄10], respectively. This gives further evidence that $\gamma \rightarrow \varepsilon$ transformation occurs in nc grains.

\section{Discussion}

The most significant feature of deformation microstructures in the $\gamma$ phase is the formation of lamellae and networks of $\varepsilon$-martensites together with deformation twins. The EDPs shown in Figs. 2-5 clearly indicate the formation of martensites and twins. This $\varepsilon$ is identified as hcp and is not to be associated with the randomly faulted $\gamma$ phase seen in Fig. 2(a), for example. The $[10 \overline{10}]_{\varepsilon}$ reflection in Fig. 2(c) will not appear if the latter description applied [16]. The sharp $\left[\begin{array}{llll}0 & 0 & 1 & 1\end{array}\right]_{\varepsilon}$ reflection, which is prohibited by the structure factor but occurs as a result of multiple diffraction, lends further support to the fact that the lattice is not randomly faulted. Streaks are also associated with reflections of $\varepsilon$-martensites containing SFs. The formation of martensites initially on individual $\{111\}_{\gamma}$ planes (Fig. 2) and subsequently on intersecting $\{111\}_{\gamma}$ planes (Fig. 3) stems from the expansion in the $[0001]_{\varepsilon}$ direction [17], as a result of which the $\gamma$ phase between the first set of parallel martensite platelets would be in a compression state. This compressive stress would lead to the formation of additional martensites on an intersecting set of $\{111\}_{\gamma}$ planes with a high shear component [17]. The $\gamma \rightarrow \varepsilon$ martensite transformation and twinning deformation continue as the strain increases and can result in ultrafine and nc grains.

The $\varepsilon$-martensites contain SFs inside them (Fig. 2(c)). The $\gamma \rightarrow \varepsilon$ transformation can be caused by the passage of $1 / 6\langle 11 \overline{2}\rangle$ Shockley partials through alternate $\{111\} \gamma$ planes. Several mechanisms have been proposed for the formation of $\varepsilon$-martensite. The pole mechanism is one of the best known [18]. However, the present result seems to provide evidence to support the mechanism proposed by Fujita and Ueda [19], namely that SFs form irregularly on $\{111\}_{\gamma}$ slip planes in the $\gamma$ phase first. More SFs are then induced on $\{111\}_{\gamma}$ planes near the original fault planes due to minimizing both the bulk free energy and the total energy of the SFs. As a result, the SFs are usually arranged on every second slip plane so that $\varepsilon$ martensites form. With this mechanism, the martensite will contain large numbers of SFs in general and diffraction spots from the $\gamma$ phase are broadened into streaks at first. In contrast, SFs are rarely induced in martensites if they are formed by a pole mechanism [19].

The deformation mechanisms of the $\gamma$ phase include the slip of dislocations by forming planar dislocation arrays, deformation twinning, and $\gamma \rightarrow \varepsilon$ transformation. All the above mechanisms may significantly contribute to work hardening during deformation. First, the planar array of dislocations may reduce ability to cross-slip, leading to enhanced strain hardening [20]. Second, twinning is highly effective in terms of enhancing the strain hardening, stemming from twin boundaries acting as strong barriers to dislocation motion (Fig. 2(b)). Third, due to the development of either the lamellae or networks of martensites, the movement of dislocations over large distances would become extremely difficult and thus, slip occurs mainly in the $\gamma$ phase, providing strain hardening. Furthermore, both $\gamma \rightarrow \varepsilon$ transformation and twinning may contribute greatly to ductility, known as the TRIP (transformation-induced plasticity) $[5,6]$ and TWIP (twinning-induced plasticity) [7] effects, respectively. When a local neck starts, strain-induced martensites and twins begin to form preferentially near the neck, leading to rapid strain hardening. Further propagation 
of this neck can be retarded, resulting in extensive, uniform elongation. The appropriate grain size distribution and introduction of second phase particles are believed to be capable of effectively enhancing ductility in nc and ultrafine grained materials [21,22]. In the present study the strain-induced $\gamma \rightarrow \varepsilon$ transformation is more than likely to be an additional candidate for improving ductility in metastable nc and ultrafine grained materials. In some respects, cobalt containing the $\gamma$ phase as a second phase can therefore be compared to other metastable materials which undergo martensite transformation and twinning on deformation, such as the cobalt alloy [5], Hadfield steel [6], and stainless steel [7,23].

\section{Conclusions}

In conclusion, $\gamma \rightarrow \varepsilon$ transformation and twinning deformation occurred successively, as the strain increased, in nanograins of $\gamma$ cobalt during surface mechanical attrition treatment. The $\varepsilon$-martensites exhibited either lamellae or intersecting networks by forming initially on individual $\{111\}_{\gamma}$ planes and subsequently on intersecting $\{111\}_{\gamma}$ planes. The martensite and twin boundaries played a significant role in blocking dislocation slipping, which is instrumental in enhancing strain hardening and ductility.

\section{Acknowledgments}

This research received backing from the National Basic Research Program of China through Grant No. 2004CB619305, National Natural Science Foundation of China Grant No. 50471086, 10472117, 50021101, the Chinese Academy of Sciences Grant No. KJCX2-
SW-L2, the NEDO International Joint Research Grant Program (01MB5), the French Ministry of Research and the Regional Council of Champagne Ardennes France (Grant 2001882, CPER EN 2040).

\section{References}

[1] Bibby MJ, Gordon Parr J. Cobalt 1963;20:111.

[2] Bibring H, Sebilleau F, Buckle CJ. Inst Metals 1958-1959;87:71.

[3] Sort J, Nogues J, Surinach S, Baro MD. Phil Mag 2003;83:439.

[4] Huang JY, Wu YQ, Ye HQ. Appl Phys Lett 1995;66:308.

[5] Singh RP, Doherty RD. Metall Trans A 1992;23A:307.

[6] Hong SH, Han YS. Scripta Metall Mater 1995;32:1489.

[7] Vercammen S, Blanpain B, De Cooman BC, Wollants. Acta Mater 2004;52:2005.

[8] Youngdahl CJ, Weertman JR, Hugo RC, Kung HH. Scripta Mater 2001;44:1475.

[9] Lu L, Shen YF, Chen XH, Qian LH, Lu K. Science 2004;304:422.

[10] Chen MW, Ma E, Hemker KJ, Sheng HW, Wang YM. Science 2003;300:1275.

[11] Liao XZ, Zhao YH, Srinivasan Zhu YT, Valiev RZ, Gunderov DV. Appl Phys Lett 2004;84:592.

[12] Rösner H, Markmann J, Weissmüller J. Phil Mag Lett 2004;84:321.

[13] Lu K, Lu J. J Mater Sci Technol 1999;15:193; Mater Sci Eng A 2004;375-377:38.

[14] Tao NR, Wu X, Sui ML, Lu J, Lu K. J Mater Res 2004;19: 1623.

[15] Wu X, Tao N, Hong Y, Liu G, Xu B, Lu J, Lu K. Acta Mater, in press.

[16] Venables JA. Phil Mag 1962;7:35.

[17] Drapier JM, Viatour P, Coutsouradis D, Harbraken L. Cobalt 1970;49:171.

[18] Houska CR, Averbach BL, Cohen M. Acta Metall 1985;33:1293.

[19] Fujita H, Ueda S. Acta Metall 1972;20:759.

[20] Karaman I, Sehitoglu H, Maier HJ, Chumlyakov YI. Acta Mater 2001;49:3919.

[21] Koch CC. Scripta Mater 2003;49:657.

[22] Wang YM, Ma E. Acta Mater 2004;52:1699.

[23] Remy L, Pineau A. Mater Sci Eng A 1997;26:123. 